Research Article

\title{
Some State-Specific Exit Probabilities in a Markov-Modulated Risk Model
}

\author{
Jingchao $\mathrm{Li} \mathbb{D}^{1,2}$ and Shuanming $\mathrm{Li}^{3}$ \\ ${ }^{1}$ College of Mathematics and Statistics, Shenzhen University, Nanhai Ave 3688, Shenzhen 518060, Guangdong, China \\ ${ }^{2}$ Shenzhen Key Laboratory of Advanced Machine Learning and Applications, Shenzhen University, Shenzhen 518060, \\ Guangdong, China \\ ${ }^{3}$ Centre for Actuarial Studies, Department of Economics, University of Melbourne, Parkville 3010, VIC, Australia
}

Correspondence should be addressed to Jingchao Li; jingchaoli@szu.edu.cn

Received 28 March 2020; Accepted 21 July 2020; Published 20 October 2020

Academic Editor: Alessandro Lo Schiavo

Copyright (c) 2020 Jingchao Li and Shuanming Li. This is an open access article distributed under the Creative Commons Attribution License, which permits unrestricted use, distribution, and reproduction in any medium, provided the original work is properly cited.

\begin{abstract}
In this paper, we study some state-specific one-sided exit probabilities in a Markov-modulated risk process including the probability that ruin occurs without or with the surplus visiting certain states; the probability that ruin occurs without or with a claim occurring in certain states; the probability that the surplus attains a target level without or with visiting certain states; and the probability that the surplus attains a target level without or with a claim occurring in certain states. We also investigate the corresponding two-sided first exit probabilities without (or with) the surplus visiting certain states or without (or with) claims occurring in certain states. All these probabilities can be expressed elegantly in terms of some modified matrix scale functions which are easily computable.
\end{abstract}

\section{Introduction}

In this paper, we consider a Markov-modulated risk model which has been studied by a number of authors since the important paper proposed by Asmussen [1]. In such a model, both the claim amounts and the claim arrivals are influenced by an external environment process which we denote by $\{J(t), t \geq 0\}$. For example, it is well known that the weather or climate conditions have impacts on automobile, property, and casualty insurance claims. This process is assumed to be a homogeneous, irreducible, and recurrent Markov process on a finite state space $E=\{1,2, \ldots, m\}$. The process has intensity matrix $\mathbf{A}=\left(\alpha_{i, j}\right)_{i, j=1}^{m}$, with $\alpha_{i, i}:=-\alpha_{i}$ for $i \in E$, and has stationary distribution $\vec{\pi}=\left(\pi_{1}\right.$, $\left.\pi_{2}, \ldots, \pi_{m}\right)$.

Let $N(t)$ denote the number of claims occurring in $(0, t]$. If $J(s)=i$ for all $s$ in a small interval $(t, t+h]$, then $N(t+$ $h)-N(t)$ represents the number of claims occurring in that interval and has a Poisson distribution with parameter $\lambda_{i}(>0)$. The process $\{N(t), t \geq 0\}$ is a Markov-modulated Poisson process, and it can also be viewed as a Poisson process with its parameter driven by the external environment process $\{J(t), t \geq 0\}$.

Given that $J(t)=i$, we assume that individual claim amounts have distribution function $F_{i}$ and probability density function $f_{i}$, with Laplace transform $\widehat{f}_{i}(s)=\int_{0}^{\infty} e^{-s x} f_{i}(x) \mathrm{d} x$ and finite mean $\mu_{i}(i \in E)$.

Then, the surplus process $\{U(t), t \geq 0\}$ in our Markovmodulated risk model is given by

$$
U(t)=u+\sum_{i=1}^{m} \int_{0}^{t} I(J(s)=i) \mathrm{d} U_{i}(s), \quad t \geq 0,
$$

where $u \geq 0$ is the initial surplus and $I$ is the indication function, and for $i \in E$, let $\left\{U_{i}(t)\right\}_{t \geq 0}$ be the surplus process in the classical risk model with premium rate $c_{i}$, Poisson parameter $\lambda_{i}$, and individual claim amount distribution $F_{i}$. The positive loading condition is also assumed to hold, i.e., 


$$
\sum_{i=1}^{m} \pi_{i}\left(c_{i}-\lambda_{i} \mu_{i}\right)>0 .
$$

We next define $T_{u}=\inf \{t \geq 0, U(t)<0\}$ to be the time of ruin, with $T_{u}=\infty$ if $U(t) \geq 0$ for all $t \geq 0$, and

$$
\psi_{i}(u)=\mathrm{P}\left(T_{u}<\infty \mid J(0)=i\right), \quad i \in E,
$$

to be the probability of ruin given that initial environment state $i$.

Let $N_{u}:=N\left(T_{u}\right)$ be the number of claims and $S_{u}:=$ $S\left(T_{u}\right)=\sum_{n=1}^{N\left(T_{u}\right)} X_{n}$ be the aggregate claims by the time of ruin, respectively, where $X_{n}$ is the amount of the $n$th claim. Now, $T_{u}, N_{u}$, and $S_{u}$ can be decomposed as

$$
\begin{aligned}
T_{u} & =\sum_{l=1}^{m} T_{u, l}, \\
N_{u} & =\sum_{l=1}^{m} N_{u, l}, \\
S_{u} & =\sum_{l=1}^{m} S_{u, l},
\end{aligned}
$$

where $T_{u, l}$ is the duration that the surplus process spends in state $l \in E$ until the time of ruin, $N_{u, l}$ is the number of claims that occur in state $l$ until the time of ruin, and $S_{u, l}$ is the aggregate claims that occur in state $l$ until the time of ruin.

For $1 \leq l_{1}<l_{2}<\cdots<l_{k} \leq m$, let $E_{k}=\left\{l_{1}, l_{2}, \ldots, l_{k}\right\} \subset E$ be a substate space. We define

$$
\begin{aligned}
T_{u, E_{k}} & =\sum_{j=1}^{k} T_{u, l_{j}}, \\
N_{u, E_{k}} & =\sum_{j=1}^{k} N_{u, l_{j}}, \\
S_{u, E_{k}} & =\sum_{j=1}^{k} S_{u, l_{j}},
\end{aligned}
$$

to be duration that the surplus spent in substate space $E_{k}=\left\{l_{1}, l_{2}, \ldots, l_{k}\right\}$, the number of claims occurred in $E_{k}$, and the aggregate claims occurred in $E_{k}$, respectively, by the time of ruin. In particular, if $E_{k}=E$, then $T_{u, E_{k}}, N_{u, E_{k}}$, and $S_{u, E_{k}}$ recover $T_{u}, N_{u}$, and $S_{u}$; if $k=1$ and $E_{k}=\{l\}$, then $T_{u, E_{k}}, N_{u, E_{k}}$, and $S_{u, E_{k}}$ simplify to $T_{u, l}, N_{u, l}$, and $S_{u, l}$, respectively.

The Markov-modulated risk models have been studied extensively in the literature. References include the studies of Asmussen et al. [2]; Bäuerle [3]; Schmidli [4]; Snoussi [5]; Lu and $\mathrm{Li}[6] ; \mathrm{Lu} \mathrm{[7]} ; \mathrm{Ng}$ and Yang [8]; and $\mathrm{Li}$ and $\mathrm{Lu}$ [9]; and $\mathrm{Li}$ and $\mathrm{Lu}[10]$. An excellent review paper of the Markovmodulated Poisson process is the paper by Fischer and Meier-Hellstern [11]. Li et al. [12] study the distributions of $T_{u}$ and $N_{u}$ for a risk model with Markovian arrival process (MAP). In the study by Li et al. [13], the one-sided exit time (time of ruin or the first hitting time), the number of claims, and the aggregate claims by the exit time are decomposed into $m$ components, respectively, according to the underlying Markov state. Joint Laplace transforms and probability generating functions of all these decomposed quantities are analyzed via matrix scale functions and matrix analysis methods. The motivation of our work is that the insurer would consider a collection of similar conditions as a whole and risk information based on the subset of states would be helpful for insurers to better understand and control their risks for a particular subset of interest and make strategic and contingency plans accordingly.

In this paper, we investigate some state-specific one-sided and two-sided first exit probabilities using several versions of the matrix scale functions for the risk model. To the best knowledge of the authors, this is the first paper to study exit probabilities of the subset of states by using different forms of the matrix scale functions. We start in Section 2 by defining these modified or dimension-reduced matrix scale functions for the Markov-modulated risk model as well as for the classical risk model. We study the probability of ruin without the surplus visiting the state-state space $E_{k}$ and the probability of ruin without claims occurring in $E_{k}$ in Sections 3 and 4 , respectively. The probability of reaching a target level without the surplus visiting $E_{k}$ and the probability of reaching a target level without claims occurring in $E_{k}$ are discussed in Sections 5 and 6, respectively. In Section 7, we study the corresponding twosided first exit probabilities without the surplus visiting $E_{k}$ or without claims occurring in $E_{k}$. Some numerical examples are demonstrated in Section 8.

\section{Notation and Preliminaries}

We define the following $m \times m$ matrices:

$$
\begin{aligned}
\mathbf{c} & =\operatorname{diag}\left(c_{1}, c_{2}, \ldots, c_{m}\right), \\
\boldsymbol{\lambda} & =\operatorname{diag}\left(\lambda_{1}, \lambda_{2}, \ldots, \lambda_{m}\right), \\
\mathbf{f}(x) & =\operatorname{diag}\left(f_{1}(x), f_{2}(x), \ldots, f_{m}(x)\right), \\
\mathbf{F}(x) & =\operatorname{diag}\left(F_{1}(x), F_{2}(x), \ldots, F_{m}(x)\right) \\
& =\mathbf{I}-\overline{\mathbf{F}}(x), \\
\mathbf{I} & =\operatorname{diag}(1,1, \ldots, 1) .
\end{aligned}
$$

Let $E_{k}=\left\{l_{1}, l_{2}, \ldots, l_{k}\right\} \subset E$ and for any $m \times m$ matrix $\mathbf{X}=\left(x_{i, j}\right)_{i, j \in E}$, we define the following four matrices:

$$
\begin{aligned}
& \mathbf{X}_{11}=\left(x_{i, j}\right)_{i, j \in E_{k}}, \\
& \mathbf{X}_{22}=\left(x_{i, j}\right)_{i, j \in E / E_{k}}, \\
& \mathbf{X}_{12}=\left(x_{i, j}\right)_{i \in E_{k}, j \in E / E_{k}}, \\
& \mathbf{X}_{21}=\left(x_{i, j}\right)_{i \in E / E_{k}, j \in E_{k}} .
\end{aligned}
$$

Here, $\mathbf{X}_{22}$ is an $(m-k) \times(m-k)$ matrix obtained by deleting the $l_{i}$-th row and the $l_{i}$-th column of $\mathbf{X}$, for $i \in E_{k}$. We remark that if $E_{k}=E$, then $\mathbf{X}_{11}=\mathbf{X}$ and $\mathbf{X}_{22}=\mathbf{X}_{12}=$ $\mathbf{X}_{21}=\varnothing$.

2.1. Matrix Scale Function and Its Modified and DimensionReduced Versions. In this section, we define a matrix-form scale function and its several modified or reduced forms which play important roles in the rest of the paper. 
Definition 1. Define $\mathbf{W}(u)$ to be a matrix function such that

$$
\begin{aligned}
\widehat{\mathbf{W}}(s) & =\int_{0}^{\infty} e^{-s u} \mathbf{W}(u) \mathrm{d} u \\
& =[\mathbf{B}(s)]^{-1},
\end{aligned}
$$

where

$$
\mathbf{B}(s)=s \mathbf{I}-\mathbf{c}^{-1}(\boldsymbol{\lambda}-\mathbf{A})+\mathbf{c}^{-1} \lambda \widehat{\mathbf{f}}(s) .
$$

Here, $\mathbf{W}(u)$ is called the matrix-form scale function for the Markov-modulated risk model with $\mathbf{W}(0)=\lim _{s \rightarrow \infty} s \hat{\mathbf{W}}(s)=\lim _{s \rightarrow \infty} s[\mathbf{B}(s)]^{-1}=\mathbf{I}$. A similar matrix-form scale function can be found in Li et al.'s study [13].

Definition 2. Define $\mathbf{W}_{11}(u)$ and $\mathbf{W}_{22}(u)$ to be a $k \times k$ matrix function and an $(m-k) \times(m-k)$ matrix function, respectively, such that

$$
\begin{aligned}
\widehat{\mathbf{W}}_{11}(s) & =\int_{0}^{\infty} e^{-s u} \mathbf{W}_{11}(u) \mathrm{d} u \\
& =\left[\mathbf{B}_{11}(s)\right]^{-1}, \\
\widehat{\mathbf{W}}_{22}(s) & =\int_{0}^{\infty} e^{-s u} \mathbf{W}_{22}(u) \mathrm{d} u \\
& =\left[\mathbf{B}_{22}(s)\right]^{-1},
\end{aligned}
$$

where

$$
\begin{aligned}
& \mathbf{B}_{11}(s)=s \mathbf{I}_{11}-\mathbf{c}_{11}^{-1}\left(\boldsymbol{\lambda}_{11}-\mathbf{A}_{11}\right)+\mathbf{c}_{11}^{-1} \boldsymbol{\lambda}_{11} \widehat{\mathbf{f}}_{11}(s), \\
& \mathbf{B}_{22}(s)=s \mathbf{I}_{22}-\mathbf{c}_{22}^{-1}\left(\boldsymbol{\lambda}_{22}-\mathbf{A}_{22}\right)+\mathbf{c}_{22}^{-1} \boldsymbol{\lambda}_{22} \widehat{\mathbf{f}}_{22}(s) .
\end{aligned}
$$

\section{Remark 1}

(1) $\mathbf{W}_{11}(u)$ and $\mathbf{W}_{22}(u)$ are called reduced matrix-form scale functions for the Markov-modulated risk model with

$$
\begin{aligned}
\mathbf{W}_{11}(0) & =\lim _{s \longrightarrow \infty} s \widehat{\mathbf{W}}_{11}(s) \\
& =\lim _{s \longrightarrow \infty} s\left[\mathbf{B}_{11}(s)\right]^{-1} \\
& =\mathbf{I}_{11},
\end{aligned}
$$

and similarly, $\mathbf{W}_{22}(0)=\mathbf{I}_{22}$.

(2) If $E_{k}=E$, then $\mathbf{W}_{11}=\mathbf{W}(u)$; if $E_{k}=\varnothing$, then $\mathbf{W}_{22}=\mathbf{W}(u)$.

(3) If $E_{k}=\{i\}$, then $\mathbf{B}_{11}(s)=s-\left(\lambda_{i}+\alpha_{i}\right) / c_{i}+\lambda_{i} \widehat{f}_{i}(s) / c_{i}$, and $\mathbf{W}_{11}(u)=\eta_{\alpha_{i}}(u)$ is the $\alpha_{i}$-scale function defined in the next section for the classical risk model.

(4) For $E_{k} \subset E$, it follows from the matrix generalization of Rouche's Theorem (De Smit [14] or Li and Ren [15]) that $\operatorname{Det}\left[\mathbf{B}_{11}(s)\right]=0$ has $k$ roots with positive real parts, say $\rho_{11,1}, \rho_{11,2}, \ldots, \rho_{11, k}$. Similarly, $\operatorname{Det}\left[\mathbf{B}_{22}(s)\right]=0$ has $m-k$ roots with positive real parts, say $\rho_{22,1}, \rho_{22,2}, \ldots, \rho_{22, m-k}$.
Definition 3. For $E_{k}=\left\{l_{1}, l_{2}, \ldots, l_{k}\right\} \subset E$, we define $\mathbf{V}\left(u, E_{k}\right)$ to be an $m \times m$ matrix function such that

$$
\begin{aligned}
\widehat{\mathbf{V}}\left(s ; E_{k}\right) & =\int_{0}^{\infty} e^{-s u} \mathbf{V}\left(u ; E_{k}\right) \mathrm{d} u \\
& =\left[\mathbf{B}\left(s ; E_{k}\right)\right]^{-1},
\end{aligned}
$$

where

$$
\mathbf{B}\left(s ; E_{k}\right)=s \mathbf{I}-\mathbf{c}^{-1}(\boldsymbol{\lambda}-\mathbf{A})+\mathbf{c}^{-1} \boldsymbol{\lambda}\left(\mathbf{I}-\mathbf{I}_{E_{k}}\right) \widehat{\mathbf{f}}(s),
$$

where $\mathbf{I}_{E_{k}}$ is an $m \times m$ diagonal matrix with the $i$-th entry being 1 , for $i \in E_{k}$, and all other entries being zeros.

\section{Remark 2}

(1) $\mathbf{V}\left(u ; E_{k}\right)$ is called a modified matrix scale function for the Markov-modulated risk model with

$$
\begin{aligned}
\mathbf{V}\left(0 ; E_{k}\right) & =\lim _{s \longrightarrow \infty} s \widehat{\mathbf{V}}\left(s ; E_{k}\right) \\
& =\lim _{s \longrightarrow \infty} s\left[\mathbf{B}\left(s ; E_{k}\right)\right]^{-1} \\
& =\mathbf{I} .
\end{aligned}
$$

(2) If $E_{k}=\varnothing$, then $\mathbf{V}(u ; \varnothing)=\mathbf{W}(u)$.

(3) For $E_{k} \subset E$, it follows from the matrix generalization of Rouche's Theorem (De Smit [14] or Li and Ren [15]) that $\operatorname{Det}\left[\mathbf{B}\left(s ; E_{k}\right)\right]=0$ has $m$ roots with positive real parts, say $\rho_{1}\left(E_{k}\right), \rho_{2}\left(E_{k}\right), \ldots, \rho_{m}\left(E_{k}\right)$.

To evaluate the matrix scale functions $\mathbf{W}(u), \mathbf{W}_{11}(u), \mathbf{W}_{22}(u)$, and $\mathbf{V}\left(u ; E_{k}\right)$, we need to make assumptions about the claim size distributions. If we assume that each $\widehat{f}_{i}(s)$ is a rational function, then we can show that each element of $\widehat{\mathbf{W}}(s)=[\mathbf{B}(s)]^{-1}=\mathbf{B}^{*}(s) / \operatorname{Det}[\mathbf{B}(s)]$ is a rational function which is readily inverted to obtain $\mathbf{W}(u)$ by partial fractions. The same are true for $\mathbf{W}_{11}(u), \mathbf{W}_{22}(u)$, and $\mathbf{V}\left(u ; E_{k}\right)$.

2.2. Scale Function in the Classical Risk Model. Let $\eta_{\alpha}(u)$ with $\eta_{\alpha}(0)=1$ be a function such that its Laplace transform $\hat{\eta}_{\alpha}(s)=\int_{0}^{\infty} e^{-s u} \eta_{\alpha}(u) \mathrm{d} u$ is given by

$$
\widehat{\eta}_{\alpha}(s)=\frac{1}{s-(\lambda+\alpha) / c+\lambda \widehat{f}(s) / c} .
$$

Remark 3

(i) $\eta_{\alpha}(u)$ is called the $\alpha$-scale function (apart from a multiplicative constant) in the classical risk model with premium rate $c$, Poisson arrival rates $\lambda$, and claim amounts density $f(x)$.

(ii) Chan et al. [16] show that $\eta_{\alpha}(u)$ has the form of 


$$
\eta_{\alpha}(u)=\frac{e^{\rho(\alpha) u}-\Psi_{\alpha}(u)}{1-\Psi_{\alpha}(0)}, \quad u \geq 0
$$

where $\Psi_{\alpha}(u)$ has three different forms and $\rho=\rho(\alpha)$ with $\rho(0)=0$ is the unique positive solution of the following generalized Lundberg's equation:

$$
\lambda+\alpha-c s=\lambda \hat{f}(s) .
$$

In particular, if $\alpha=0, \Psi_{0}(u)=\psi(u)$ simplifies to the ruin probability for the classical risk model and $\eta_{0}(u)=(1-\psi(u)) /(1-\psi(0))$.

(iii) If we replace $c$ by $c_{k}$, replace $\lambda$ by $\lambda_{k}$, replace $f(x)$ by $f_{k}(x)$, and replace $\alpha$ by $\alpha_{k}$, then we write $\eta_{\alpha}(u)$ as $\eta_{\alpha_{k}}(u)$ and write $\rho=\rho(\alpha)$ as $\rho_{k}=\rho_{k}\left(\alpha_{k}\right)$, for $k=1,2, \ldots, m$.

\section{Probability of Ruin without the Surplus Visiting States in $E_{k}$}

First, we define for $i \in E$,

$$
\begin{aligned}
\psi_{i}\left(u ; E_{k}\right) & =P\left(T_{u}<\infty, J(t) \notin E_{k}, 0 \leq t \leq T_{u} \mid J(0)=i\right) \\
& =P\left(T_{u}<\infty, T_{u, E_{k}}=0 \mid J(0)=i\right),
\end{aligned}
$$

to be the probability of ruin without the external environmental Markov process visiting the substate space $E_{k}$ prior to ruin.

\section{Remark 4}

(1) It follows from the definition above that $\psi_{i}\left(u ; E_{k}\right)=$ 0 if $i \in E_{k}$.

(2) $\psi_{i}(u ;\{k\})$ is the probability of ruin without the surplus visiting state $k$, for $k \neq i$, given $J(0)=i$.

(3) $\psi_{i}\left(u ; E \backslash\left\{E_{k}\right\}\right)$ is the probability of ruin with the surplus ever visiting every state in $E_{k}$.

(4) $\psi_{i}(u ; E \backslash\{i\})$ is the probability of ruin with the surplus only visiting the initial state $i$.

(5) If $E_{k}=\varnothing$, then $\psi_{i}(u ; \varnothing)=\psi_{i}(u)$ is the probability of ruin given that the initial state is $i$.

Conditioning on the events that may occur in an infinitesimal interval, we can obtain the following IDE for $\psi_{i}\left(u ; E_{k}\right)$ :

$$
\begin{aligned}
c_{i} \psi_{i}^{\prime}\left(u ; E_{k}\right)= & \lambda_{i} \psi_{i}\left(u ; E_{k}\right)-\lambda_{i} \int_{0}^{u} \psi_{i}\left(u-x ; E_{k}\right) f_{i}(x) \mathrm{d} x \\
& -\lambda_{i} \bar{F}_{i}(u)-\sum_{j=1, j \notin E_{k}}^{m} \alpha_{i, j} \psi_{j}\left(u ; E_{k}\right), \quad i \in E \backslash\left\{E_{k}\right\} .
\end{aligned}
$$

Let $\vec{\psi}\left(u ; E_{k}\right)=\left(\psi_{i}\left(u ; E_{k}\right), i \in E \backslash\left\{E_{k}\right\}\right)^{\top}$ be an $(m-k) \times$ 1 column vector. Equation (20) can be rewritten in matrix form as

$$
\begin{aligned}
\vec{\psi}^{\prime}\left(u ; E_{k}\right) & =\mathbf{c}_{22}^{-1}\left(\boldsymbol{\lambda}_{22}-\mathbf{A}_{22}\right) \vec{\psi}\left(u ; E_{k}\right) \\
& =-\mathbf{c}_{22}^{-1} \boldsymbol{\lambda}_{22}\left[\int_{0}^{u} \mathbf{f}_{22}(x) \vec{\psi}\left(u-x ; E_{k}\right) \mathrm{d} x+\overline{\mathbf{F}}_{22}(u) \overrightarrow{1}\right] .
\end{aligned}
$$

Denote $\widehat{\vec{\psi}}\left(s ; E_{k}\right)=\int_{0}^{\infty} e^{-s u} \vec{\psi}\left(u ; E_{k}\right) \mathrm{d} u$. Taking Laplace transforms of both sides of equation (21) gives

$$
\mathbf{B}_{22}(s) \hat{\vec{\psi}}\left(s ; E_{k}\right)=\vec{\psi}\left(0 ; E_{k}\right)-\mathbf{c}_{22}^{-1} \boldsymbol{\lambda}_{22} \widehat{\overline{\mathbf{F}}}_{22}(s) \overrightarrow{1},
$$

where $\mathbf{B}_{22}(s)=s \mathbf{I}_{22}-\mathbf{c}_{22}^{-1}\left(\lambda_{22}-\mathbf{A}_{22}\right)+\mathbf{c}_{22}^{-1} \lambda_{22} \widehat{\mathbf{f}}_{22}(s)$. Use the same arguments as in the study by Li et al. [13] to find $\vec{\psi}\left(0 ; E_{k}\right)$. Let $\overrightarrow{\mathbf{q}}_{22, i}^{\top}$ be a $1 \times(m-k)$ row vector such that $\overrightarrow{\mathbf{q}}_{22, i}^{\top} \mathbf{B}_{22}\left(\rho_{22, i}\right)=\overrightarrow{0}^{\top}$, for $i=1,2, \ldots, m-k$. Setting $s=\rho_{22, i}$ and left-multiplying both sides of (22) by the vector $\overrightarrow{\mathbf{q}}_{22, i}^{\top}$, we have

$$
\begin{array}{r}
\overrightarrow{0}^{\top}=\overrightarrow{\mathbf{q}}_{22, i}^{\top} \mathbf{B}_{22}\left(\rho_{22, i}\right) \hat{\vec{\psi}}\left(\rho_{22, i} ; E_{k}\right) \\
=\overrightarrow{\mathbf{q}}_{22, i}^{\top} \vec{\psi}\left(0 ; E_{k}\right)-\overrightarrow{\mathbf{q}}_{22, i}^{\top} \mathbf{c}_{22}^{-1} \boldsymbol{\lambda}_{22} \hat{\overline{\mathbf{F}}}_{22}\left(\rho_{22, i}\right) \overrightarrow{1}, \\
i=1,2, \ldots, m-k .
\end{array}
$$

Since $\mathbf{c}_{22}, \lambda_{22}$, and $\overline{\mathbf{F}}_{22}(u)$ are all diagonal matrixes,

$$
\begin{aligned}
\overrightarrow{\mathbf{q}}_{22, i}^{\top} \vec{\psi}\left(0 ; E_{k}\right) & =\overrightarrow{\mathbf{q}}_{22, i}^{\top} \mathbf{c}_{22}^{-1} \boldsymbol{\lambda}_{22} \widehat{\overline{\mathbf{F}}}_{22}\left(\rho_{22, i}\right) \overrightarrow{1} \\
& =\overrightarrow{\mathbf{q}}_{22, i}^{\top} \mathbf{c}_{22}^{-1} \boldsymbol{\lambda}_{22} \int_{0}^{\infty} e^{-\rho_{22, i} u} \overline{\mathbf{F}}_{22}(x) \mathrm{d} x \overrightarrow{\mathbf{1}} \\
& =\int_{0}^{\infty} e^{-\rho_{22, i} u} \overrightarrow{\mathbf{q}}_{22, i}^{\top} \overline{\mathbf{F}}_{22}(x) \mathrm{d} x \mathbf{c}_{22}^{-1} \boldsymbol{\lambda}_{22} \overrightarrow{\mathrm{l}} .
\end{aligned}
$$

In matrix form, we have

$$
\vec{\psi}\left(0 ; E_{k}\right)=\int_{0}^{\infty} e^{-x \boldsymbol{\Theta}_{22}} \overline{\mathbf{F}}_{22}(x) d x \mathbf{c}_{22}^{-1} \boldsymbol{\lambda}_{22} \overrightarrow{1},
$$

where $\boldsymbol{\Theta}_{22}=\mathbf{q}_{22}^{-1} \rho_{22} \mathbf{q}_{22}, \boldsymbol{\rho}_{22}=\operatorname{diag}\left(\rho_{22,1}, \rho_{22,2}, \ldots, \rho_{22, m-k}\right)$, and

$$
\mathbf{q}_{22}=\left(\overrightarrow{\mathbf{q}}_{22,1}, \overrightarrow{\mathbf{q}}_{22,2}, \ldots, \overrightarrow{\mathbf{q}}_{22, m-k}\right)^{\top},
$$

with $\overrightarrow{\mathbf{q}}_{22, i}^{\top}$ being a $1 \times(m-k)$ row vector such that $\overrightarrow{\mathbf{q}}_{22, i}^{\top} \mathbf{B}_{22}\left(\rho_{22, i}\right)=\overrightarrow{0}^{\top}$, for $i=1,2, \ldots, m-k$.

3.1. Explicit Expression in terms of the Dimension-Reduced Matrix Scale Function. Rearranging (22) gives

$$
\widehat{\vec{\psi}}\left(s ; E_{k}\right)=\left[\mathbf{B}_{22}(s)\right]^{-1}\left[\vec{\psi}\left(0 ; E_{k}\right)-\mathbf{c}_{22}^{-1} \boldsymbol{\lambda}_{22} \hat{\overline{\mathbf{F}}}_{22}(s) \overrightarrow{1}\right],
$$

as the Laplace transform of $\mathbf{W}_{22}(u)$ is $\left[\mathbf{B}_{22}(s)\right]^{-1}$; then, by inverting (27), it gives 


$$
\begin{aligned}
\vec{\psi}\left(u ; E_{k}\right)= & \mathbf{W}_{22}(u) \vec{\psi}\left(0 ; E_{k}\right) \\
& -\int_{0}^{u} \mathbf{W}_{22}(u-x) \overline{\mathbf{F}}_{22}(x) \mathrm{d} x \mathbf{c}_{22}^{-1} \boldsymbol{\lambda}_{22} \overrightarrow{1} \\
= & \int_{0}^{\infty} \mathbf{R}_{22}(u, x) \overline{\mathbf{F}}_{22}(x) \mathrm{d} x \mathbf{c}_{22}^{-1} \boldsymbol{\lambda}_{22} \overrightarrow{1}, \quad u>0
\end{aligned}
$$

where $\mathbf{W}_{22}(u)$ is the modified matrix version of the scale function defined in Section 2 and

$$
\mathbf{R}_{22}(u, x)= \begin{cases}\mathbf{W}_{22}(u) e^{-\Theta_{22} x}-\mathbf{W}_{22}(u-x), & 0 \leq x \leq u \\ \mathbf{W}_{22}(u) e^{-\boldsymbol{\Theta}_{22} x}, & x>u\end{cases}
$$

\section{Remark 5}

(1) Following the same reasoning, we have

$$
\begin{aligned}
& \vec{\psi}\left(u ; E \backslash E_{k}\right)=\int_{0}^{\infty} \mathbf{R}_{11}(u, x) \overline{\mathbf{F}}_{11}(x) \mathrm{d} x \mathbf{c}_{11}^{-1} \lambda_{11} \overrightarrow{1}, \quad u>0 \\
& \vec{\psi}\left(0 ; E \backslash E_{k}\right)=\int_{0}^{\infty} e^{-x \Theta_{11}} \overline{\mathbf{F}}_{11}(x) \mathrm{d} x \mathbf{c}_{11}^{-1} \lambda_{11} \overrightarrow{1}
\end{aligned}
$$

where $\mathbf{W}_{11}(u)$ is the dimension-reduced matrix scale function defined in Section 2, and

$$
\begin{aligned}
\mathbf{R}_{11}(u, x) & = \begin{cases}\mathbf{W}_{11}(u) e^{-\Theta_{11} x}-\mathbf{W}_{11}(u-x), & 0 \leq x \leq u, \\
\mathbf{W}_{11}(u) e^{-\Theta_{11} x}, & x>u,\end{cases} \\
\boldsymbol{\Theta}_{11} & =\mathbf{q}_{11}^{-1} \rho_{11} \mathbf{q}_{11}, \\
\rho_{11} & =\operatorname{diag}\left(\rho_{11,1}, \rho_{11,2}, \ldots, \rho_{11, k}\right), \\
\mathbf{q}_{11} & =\left(\overrightarrow{\mathbf{q}}_{11,1}, \overrightarrow{\mathbf{q}}_{11,2}, \ldots, \overrightarrow{\mathbf{q}}_{11, m-k}\right)^{\top},
\end{aligned}
$$

with $\overrightarrow{\mathbf{q}}_{11, i}^{\top}$ being a $1 \times k$ row vector such that $\overrightarrow{\mathbf{q}}_{11, i}^{\top} \mathbf{B}_{11}\left(\rho_{11, i}\right)=\overrightarrow{0}^{\top}$, for $i=1,2, \ldots, k$.

(2) In particular, we have

$$
\psi_{i}(0 ; E \backslash\{i\})=\frac{\lambda_{i}}{c_{i}} \int_{0}^{\infty} e^{-\rho_{i} u} \bar{F}_{i}(u) \mathrm{d} u,
$$

where $\rho_{i}$ is the unique positive solution of

$$
\lambda_{i}+\alpha_{i}-c_{i} s=\lambda_{i} \widehat{f}_{i}(s), \quad i=1,2, \ldots, m .
$$

Equation (30) reduces to the following for $u>0$ :

$$
\begin{aligned}
\psi_{i}(u ; E \backslash\{i\})= & \eta_{\alpha_{i}}(u) \psi_{i}(0 ; E \backslash\{i\}) \\
& -\frac{\lambda_{i}}{c_{i}} \int_{0}^{u} \eta_{\alpha_{i}}(u-x) \bar{F}_{i}(x) \mathrm{d} x, \quad i=1,2, \ldots, m,
\end{aligned}
$$

where $\psi_{i}(u ; E \backslash\{i\})$ is the probability of ruin with the surplus only visiting the initial state $i$.

3.2. Exponential Claim Distributions. We assume that $f_{i}(x)=\boldsymbol{\beta}_{i} e^{-\boldsymbol{\beta}_{i} x}$ with $\boldsymbol{\beta}_{i}>0, x>0, i \in E$, i.e., $\mathbf{f}(x)=\boldsymbol{\beta} e^{-\boldsymbol{\beta} x}$, with $\boldsymbol{\beta}=\operatorname{diag}\left(\boldsymbol{\beta}_{1}, \boldsymbol{\beta}_{2}, \ldots, \boldsymbol{\beta}_{m}\right)$. Differentiating equation (21), we obtain a 2 nd-order matrix differential equation as follows:

$$
\begin{aligned}
& \overrightarrow{\boldsymbol{\psi}}^{\prime \prime}\left(u ; E_{k}\right)+\left[\boldsymbol{\beta}_{22}-\mathbf{c}_{22}^{-1}\left(\boldsymbol{\lambda}_{22}-\mathbf{A}_{22}\right)\right] \overrightarrow{\boldsymbol{\psi}}^{\prime}\left(u ; E_{k}\right) \\
& +\mathbf{c}_{22}^{-1} \boldsymbol{\beta}_{22} \mathbf{A}_{22} \vec{\psi}\left(u ; E_{k}\right)=\overrightarrow{0},
\end{aligned}
$$

with $\lim _{u \longrightarrow \infty} \vec{\psi}\left(u ; E_{k}\right)=\overrightarrow{0} \quad$ and $\quad \lim _{u \rightarrow 0} \vec{\psi}(u$; $\left.E_{k}\right)=\vec{\psi}\left(0 ; E_{k}\right)$. The solution to this matrix differential equation is

$$
\vec{\psi}\left(u ; E_{k}\right)=e^{-\mathrm{R} u} \vec{\psi}\left(0 ; E_{k}\right), \quad u>0,
$$

where $\vec{\psi}\left(0 ; E_{k}\right)$ is given in $(25)$ and $\mathbf{R}$ is an $(m-k) \times(m-$ $k$ ) matrix satisfying the following equation:

$$
\mathbf{R}^{2}-\left[\boldsymbol{\beta}_{22}-\mathbf{c}_{22}^{-1}\left(\boldsymbol{\lambda}_{22}-\mathbf{A}_{22}\right)\right] \mathbf{R}+\mathbf{c}_{22}^{-1} \boldsymbol{\beta}_{22} \mathbf{A}_{22}=0 .
$$

\section{Remark 6}

(i) The solution to (37) is not unique, and the one whose eigenvalues have positive real parts is our $\mathbf{R}$

(ii) A study of approaches to solve quadratic matrix equations is conducted in Higham and Kim [17]

In particular, if $m=2$ and $E_{1}=\{2\}$, then it follows from equation (36) that

$$
\psi_{1}(u ;\{2\})=e^{-R_{1} u} \psi_{1}(0 ;\{2\}), \quad u>0
$$

where $R_{1}$ is the positive solution of the following quadratic equation:

$$
c_{1} s^{2}-\left[c_{1} \beta_{1}-\left(\lambda_{1}+\alpha_{1}\right)\right] s-\beta_{1} \alpha_{1}=0,
$$

and $\psi_{1}(0 ;\{2\})$ is given by $(32)$ as follows:

$$
\begin{aligned}
\psi_{1}(0 ;\{2\}) & =\frac{\lambda_{1}}{c_{1}} \int_{0}^{\infty} e^{-\rho_{1} x} \bar{F}_{1}(x) \mathrm{d} x \\
& =\frac{\lambda_{1}}{c_{1}} \int_{0}^{\infty} e^{-\rho_{1} x} e^{-\beta_{1} x} \mathrm{~d} x \\
& =\frac{\lambda_{1}}{c_{1}\left(\rho_{1}+\beta_{1}\right)},
\end{aligned}
$$

with $\rho_{1}$ satisfying equation $\lambda_{1}+\alpha_{1}-c_{1} s=\lambda_{1} \widehat{f}_{1}(s)$. We comment that $-\rho_{1}$ is the negative solution to equation (39).

\section{Probability of Ruin without a Claim Occurring in States in $E_{k}$}

For $E_{k}=\left\{l_{1}, l_{2}, \ldots, l_{k}\right\}$ and for $i \in E$, let

$$
\chi_{i}\left(u ; E_{k}\right)=\mathrm{P}\left(T_{u}<\infty, N_{u, E_{k}}=0 \mid J(0)=i\right)
$$


be the probability of ruin without a claim occurring in $E_{k}$ by the time of ruin given that $J(0)=i$.

\section{Remark 7}

(1) $\chi_{i}(u ;\{k\})$ is the probability of ruin without a claim occurring in state $k$, for $k \in E$, given $J(0)=i$

(2) $\chi_{i}\left(u ; E \backslash\left\{E_{k}\right\}\right)$ is the probability of ruin with claims occurring in states in $E_{k}$

(3) $\chi_{i}(u ; E \backslash\{i\})$ is the probability of ruin with claims only occurring when the process is in the state $i$

(4) If $E_{k}=\varnothing$, then $\chi_{i}(u ; \varnothing)$ recovers the ruin probability $\psi_{i}(u)$

(5) Note that $\chi_{i}\left(u ; E_{k}\right)-\psi_{i}\left(u ; E_{k}\right)$ is the probability of ruin with the surplus visiting $E_{k}$, but without claims occurring in $E_{k}$

Conditioning on the events that may occur in an infinitesimal interval, we can obtain the following IDE for $\chi_{i}\left(u ; E_{k}\right)$ :

$$
\begin{aligned}
c_{i} \chi_{i}^{\prime}\left(u ; E_{k}\right)= & \lambda_{i} \chi_{i}\left(u ; E_{k}\right)-\sum_{j=1}^{m} \alpha_{i, j} \chi_{j}\left(u ; E_{k}\right) \\
& -\lambda_{i} \int_{0}^{u} \chi_{i}\left(u-x ; E_{k}\right) f_{i}(x) \mathrm{d} x-\lambda_{i} \bar{F}_{i}(u), \quad i \notin E_{k}, \\
c_{i} \chi_{i}^{\prime}\left(u ; E_{k}\right)= & \lambda_{i} \chi_{i}\left(u ; E_{k}\right)-\sum_{j=1}^{m} \alpha_{i, j} \chi_{j}\left(u ; E_{k}\right), \quad i \in E_{k} .
\end{aligned}
$$

Let $\vec{\chi}\left(u ; E_{k}\right)=\left(\chi_{i}\left(u ; E_{k}\right) ; i \in E\right)^{\top}$ be an $m \times 1$ column vector. In the matrix form, we have

$$
\begin{aligned}
\vec{\chi}^{\prime}\left(u ; E_{k}\right)= & \mathbf{c}^{-1}(\boldsymbol{\lambda}-\mathbf{A}) \vec{\chi}\left(u ; E_{k}\right)-\mathbf{c}^{-1} \boldsymbol{\lambda}\left(\mathbf{I}-\mathbf{I}_{E_{k}}\right) \\
& \cdot \int_{0}^{u} \mathbf{f}(x) \vec{\chi}\left(u-x ; E_{k}\right) \mathrm{d} x-\mathbf{c}^{-1} \boldsymbol{\lambda}\left(\mathbf{I}-\mathbf{I}_{E_{k}}\right) \overline{\mathbf{F}}(u) \overrightarrow{1} .
\end{aligned}
$$

Taking Laplace transforms of both sides of (43), we have

$$
\mathbf{B}\left(s ; E_{k}\right) \widehat{\vec{\chi}}\left(s ; E_{k}\right)=\vec{\chi}\left(0 ; E_{k}\right)-\mathbf{c}^{-1} \boldsymbol{\lambda}\left(\mathbf{I}-\mathbf{I}_{E_{k}}\right) \hat{\overline{\mathbf{F}}}(s) \overrightarrow{1},
$$

where $\mathbf{B}\left(s ; E_{k}\right)=s \mathbf{I}-\mathbf{c}^{-1}(\lambda-\mathbf{A})+\mathbf{c}^{-1} \lambda\left(\mathbf{I}-\mathbf{I}_{E_{k}}\right) \widehat{\mathbf{f}}(s)$. Then,

$$
\begin{aligned}
\vec{\chi}\left(0 ; E_{k}\right)= & \int_{0}^{\infty} e^{-x \Theta_{E_{k}}} \overline{\mathbf{F}}(x) \mathrm{d} x \mathbf{c}^{-1} \lambda\left(\mathbf{I}-\mathbf{I}_{E_{k}}\right) \overrightarrow{1}, \\
\vec{\chi}\left(u ; E_{k}\right)= & \mathbf{V}\left(u ; E_{k}\right) \vec{\chi}\left(0 ; E_{k}\right) \\
& -\int_{0}^{u} \mathbf{V}\left(u-x ; E_{k}\right) \overline{\mathbf{F}}(x) \mathrm{d} x \mathbf{c}^{-1} \lambda\left(\mathbf{I}-\mathbf{I}_{E_{k}}\right) \overrightarrow{1} \\
= & \int_{0}^{\infty} \mathbf{R}\left(u, x ; E_{k}\right) \overline{\mathbf{F}}(x) \mathrm{d} x \mathbf{c}^{-1} \lambda\left(\mathbf{I}-\mathbf{I}_{E_{k}}\right) \overrightarrow{1},
\end{aligned}
$$

where $\mathbf{V}\left(u ; E_{k}\right)$ is a modified matrix scale function defined in Section 2. And,

$$
\begin{aligned}
\mathbf{R}\left(u, x ; E_{k}\right) & = \begin{cases}\mathbf{V}\left(u ; E_{k}\right) e^{-x \boldsymbol{\Theta}_{E_{k}}}-\mathbf{V}\left(u-x ; E_{k}\right), & 0 \leq x \leq u, \\
\mathbf{V}\left(u ; E_{k}\right) e^{-x \boldsymbol{\Theta}_{E_{k}},} & x>u,\end{cases} \\
\boldsymbol{\Theta}_{E_{k}} & =\left[\mathbf{Q}_{E_{k}}\right]^{-1} \operatorname{diag}\left(\rho_{1}\left(E_{k}\right), \rho_{2}\left(E_{k}\right), \ldots, \rho_{m}\left(E_{k}\right)\right) \mathbf{Q}_{E_{k}}, \\
\mathbf{Q}_{E_{k}} & =\left(\overrightarrow{\mathbf{q}}_{E_{k},}, \overrightarrow{\mathbf{q}}_{E_{k}, 2}, \ldots, \overrightarrow{\mathbf{q}}_{E_{k}, m}\right)^{\top},
\end{aligned}
$$

with $\overrightarrow{\mathbf{q}}_{E_{k}, i}^{\top}$ being a $1 \times$ mrow vector such that $\overrightarrow{\mathbf{q}}_{E_{k}, i}^{\top} \mathbf{B}\left(\rho_{i}\right.$ $\left.\left(E_{k}\right) ; E_{k}\right)=\overrightarrow{0}^{\top}$, for $i=1,2, \ldots, m$.

If we assume claim amounts are exponentially distributed, i.e., $\mathbf{f}(x)=\boldsymbol{\beta} e^{-\boldsymbol{\beta} x}$, with $\boldsymbol{\beta}=\operatorname{diag}\left(\boldsymbol{\beta}_{1}, \boldsymbol{\beta}_{2}, \ldots, \boldsymbol{\beta}_{m}\right)$, then by differentiating equation (43), we can obtain a 2 nd-order matrix differential equation for $\vec{\chi}\left(u ; E_{k}\right)$ whose solution is given by

$$
\vec{\chi}\left(u ; E_{k}\right)=e^{-\Delta u} \vec{\chi}\left(0 ; E_{k}\right), \quad u>0,
$$

where $\Delta$ is the solution to the following quadratic matrix equation:

$$
\boldsymbol{\Delta}^{2}-\left[\boldsymbol{\beta}-\mathbf{c}^{-1}(\boldsymbol{\lambda}-\mathbf{A})\right] \boldsymbol{\Delta}+\mathbf{c}^{-1} \boldsymbol{\beta}\left(\lambda \mathbf{I}_{E_{k}}-\mathbf{A}\right)=0 .
$$

We remark that the solutions to the equation above is not unique and the one with positive eigenvalues is our $\Delta$.

\section{Probability of Hitting a Target Level without the Surplus Ever Visiting States in $E_{k}$}

For $u \leq b$, let $T_{u}^{b}=\min (t \geq 0, U(t)=b)$ be the first time when the surplus reaches level $b$. We define

$$
\begin{array}{r}
\psi_{i, j}\left(u, b ; E_{k}\right)=\mathrm{P}\left(J(t) \notin E_{k}, J\left(T_{u}^{b}\right)=j, 0 \leq t \leq T_{u}^{b} \mid J(0)=i\right), \\
i, j \notin E_{k},
\end{array}
$$

to be the probability of hitting a target level $b$ at state $j$ without the surplus ever visiting substate space $E_{k}$, given that the initial state is $i \notin E_{k}$.

Remark 8

(1) $\psi_{i, j}(u, b ; \varnothing)$ is the probability of hitting a target level $b$ at state $j$ given that the initial state is $i$

(2) $\psi_{i, j}(u, b ; E \backslash\{i, j\})$ is the probability of hitting a target level $b$ at state $j$ from the initial state $i$ without the surplus visiting any other states

By conditioning on the events that may occur in an infinitesimal interval, we obtain the following IDE:

$$
\begin{aligned}
\psi^{\prime}\left(u, b ; E_{k}\right)= & \mathbf{c}_{22}^{-1}\left(\boldsymbol{\lambda}_{22}-\mathbf{A}_{22}\right) \psi\left(u, b ; E_{k}\right) \\
& -\mathbf{c}_{22}^{-1} \boldsymbol{\lambda}_{22} \int_{0}^{\infty} \mathbf{f}_{22}(y) \psi\left(u-y ; b ; E_{k}\right) \mathrm{d} x,
\end{aligned}
$$

where $\psi\left(u, b ; E_{k}\right)=\left(\psi_{i, j}\left(u, b ; E_{k}\right) ; i, j \in E \backslash\left\{E_{k}\right\}\right)$ is an $(m-$ $k) \times(m-k)$ matrix.

The following three properties are satisfied by $\psi_{i, j}$ : 
Property 1: $\psi_{i, j}\left(b, b ; E_{k}\right)=I(i=j)$

Property $2: \psi_{i, j}\left(u, b ; E_{k}\right)$ is a function of $(b-u)$

Property 3: $\psi_{i, j}\left(u, b ; E_{k}\right)=\sum_{l \notin E_{k}} \psi_{i, l}\left(u, b_{1} ; E_{k}\right) \psi_{l, j}\left(b_{1}, b\right.$; $\left.E_{k}\right)$, for $u \leq b_{1} \leq b$

This shows that $\psi\left(u, b ; E_{k}\right)$ must have the following form:

$$
\psi\left(u, b ; E_{k}\right)=e^{-\Gamma_{22}(b-u)}, \quad u \leq b,
$$

where $\Gamma_{22}$ is an $(m-k) \times(m-k)$ matrix and all of its eigenvalues must have positive real parts. It can be showed by substituting (51) into (50) that $\Gamma_{22}$ satisfies a matrix equation as follows:

$$
\Gamma_{22}+\mathbf{c}_{22}^{-1} \boldsymbol{\lambda}_{22} \int_{0}^{\infty} \mathbf{f}_{22}(x) e^{-\Gamma_{22} x} \mathrm{~d} x-\mathbf{c}_{22}^{-1}\left(\boldsymbol{\lambda}_{22}-\mathbf{A}_{22}\right)=0,
$$

with the following solution:

$$
\Gamma_{22}=\mathbf{H}_{22} \rho_{22} \mathbf{H}_{22}^{-1} \text {, }
$$

where $\mathbf{H}_{22}=\left(\overrightarrow{\mathbf{h}}_{22,1}, \overrightarrow{\mathbf{h}}_{22,2}, \ldots, \overrightarrow{\mathbf{h}}_{22, m-k}\right), \boldsymbol{\rho}_{22}=\operatorname{diag}\left(\rho_{22,1}\right.$, $\left.\rho_{22,2}, \ldots, \rho_{22, m-k}\right)$, and $\rho_{22,1}, \rho_{22,2}, \ldots, \rho_{22, m-k}$ are the $(m-k)$ distinct roots of $\operatorname{Det}\left[\mathbf{B}_{22}(s)\right]=0$. The column vector $\overrightarrow{\mathbf{h}}_{22, i}$ is that $\mathbf{B}_{22}\left(\rho_{22, i}\right) \overrightarrow{\mathbf{h}}_{22, i}=\overrightarrow{0}$.

In particular, for $E_{k}=E \backslash\{i\}$, the result in (51) simplifies to

$$
\psi_{i, i}(u, b ; E \backslash\{i\})=e^{-\rho_{i}(b-u)}, \quad u \leq b,
$$

which is the probability of hitting a target level $b$ at state $i$ from the initial state $i$ without the surplus visiting any other states.

\section{Probability of Hitting a Target Level without a Claim Occurring in States in $E_{k}$}

For $u \leq b$, define $\chi_{i, j}\left(u, b ; E_{k}\right)$ to be the probability of hitting a target level $b$ at state $j \in E$ without a claim occurring in states in $E_{k}$, given that the initial state is $i \in E$.

\section{Remark 9}

(1) $\chi_{i, j}(u, b ; \varnothing)=\psi_{i, j}(u, b ; \varnothing)$ is the probability of hitting a target level $b$ at state $j$ given that the initial state is $i$

(2) $\chi_{i, j}(u, b ; E)$ is the probability of hitting a target level $b$ at state $j$ from the initial state $i$ without any claim occurring

(3) $\chi_{i, j}(u, b ; E \backslash\{k\})$ is the probability of hitting a target level $b$ at state $j$ from the initial state $i$ with claims occurring only in state $k$

Using the same arguments as in the previous section, we have for $u \leq b$,

$$
\begin{aligned}
c_{i} \chi_{i, j}^{\prime}\left(u, b ; E_{k}\right)= & \lambda_{i} \chi_{i, j}\left(u, b ; E_{k}\right)-\sum_{k=1}^{m} \alpha_{i, k} \chi_{k, j}\left(u, b ; E_{k}\right) \\
& -\lambda_{i} \int_{0}^{\infty} \chi_{i, j}\left(u-x, b ; E_{k}\right) f_{i}(x) \mathrm{d} x, \quad i \notin E_{k} \\
c_{i} \chi_{i, j}^{\prime}\left(u, b ; E_{k}\right)= & \lambda_{i} \chi_{i, j}\left(u, b ; E_{k}\right)-\sum_{k=1}^{m} \alpha_{i, k} \chi_{k, j}\left(u, b ; E_{k}\right), \quad i \in E_{k} .
\end{aligned}
$$

This is written in matrix form as

$$
\begin{aligned}
\chi^{\prime}\left(u, b ; E_{k}\right) & =\mathbf{c}^{-1}(\boldsymbol{\lambda}-\mathbf{A}) \chi\left(u, b ; E_{k}\right) \\
& =-\mathbf{c}^{-1} \boldsymbol{\lambda}\left[\mathbf{I}-\mathbf{I}_{E_{k}}\right] \int_{0}^{\infty} \mathbf{f}(x) \chi\left(u-x ; b ; E_{k}\right) \mathrm{d} x,
\end{aligned}
$$

where $\chi\left(u, b ; E_{k}\right)=\left(\chi_{i, j}\left(u, b ; E_{k}\right) ; i, j \in E\right)$ is an $m \times m$ matrix. Clearly, $\chi_{i, j}\left(u, b ; E_{k}\right)$ has the same three properties as for $\psi_{i, j}\left(u, b ; E_{k}\right)$ stated in Section 5 , and we have

$$
\chi\left(u, b ; E_{k}\right)=e^{-\Gamma_{E_{k}}(b-u)}, \quad u \leq b,
$$

where $\Gamma_{E_{k}}$ is an $m \times m$ matrix and all of its eigenvalues must have positive real parts and $\Gamma_{E_{k}}$ satisfies an matrix equation with the following solution:

$$
\Gamma_{E_{k}}=\mathbf{H}_{E_{k}} \rho\left(E_{k}\right) \mathbf{H}_{E_{k}}^{-1},
$$

where $\quad \mathbf{H}_{E_{k}}=\left(\overrightarrow{\mathbf{h}}_{E_{k}, 1}, \overrightarrow{\mathbf{h}}_{E_{k}, 2}, \ldots, \overrightarrow{\mathbf{h}}_{E_{k}, m}\right), \quad \rho\left(E_{k}\right)=\operatorname{diag}$ $\left(\rho_{1}\left(E_{k}\right), \rho_{2}\left(E_{k}\right), \ldots, \rho_{m}\left(E_{k}\right)\right), \quad$ and $\rho_{1}\left(E_{k}\right), \rho_{2}\left(E_{k}\right), \ldots$, $\rho_{m}\left(E_{k}\right)$ are the $m$ distinct roots of $\operatorname{Det}\left[\mathbf{B}\left(s ; E_{k}\right)\right]=0$. The column vector $\overrightarrow{\mathbf{h}}_{E_{k}, i}$ is that $\mathbf{B}\left(\rho_{i}\left(E_{k}\right) ; E_{k}\right) \overrightarrow{\mathbf{h}}_{E_{k}, i}=\overrightarrow{0}$.

In particular, when $E_{k}=E$, then equations (55) and (56) simplify to

$$
\chi^{\prime}(u, b ; E)=\mathbf{c}^{-1}(\boldsymbol{\lambda}-\mathbf{A}) \chi(u, b ; E),
$$

with the following solution:

$$
\chi(u, b ; E)=e^{\mathbf{c}^{-1}(\mathbf{A}-\lambda)(b-u)}, \quad u \leq b .
$$

\section{Two-Sided First Exit Probabilities}

In Sections 3-6, we have studied the one-sided exit probability without the surplus visiting states in $E_{k}$ or without a claim occurring in states in $E_{k}$. In this section, we aim to investigate the corresponding two-sided first exit probabilities.

7.1. Probability of Hitting a Target Level prior to Ruin without the Process Visiting States in $E_{k}$. For $0 \leq u \leq b$ and $i, j \in E$, we define 


$$
\begin{array}{r}
\xi_{i, j}\left(u, b ; E_{k}\right)=\mathrm{P}\left(T_{u}^{b}<T_{u}, J\left(T_{u}^{b}\right)=j,\right. \\
\left.J(t) \notin E_{k}, 0 \leq t<T_{u}^{b} \mid J(0)=i\right),
\end{array}
$$

to be the probability of reaching $b$ in state $j$ with neither ruin occurring nor the process visiting states in $E_{k}$, given that the initial state is $i$. Clearly, we have the following cases:

(1) $\xi_{i, j}\left(u, b ; E_{k}\right)=0$, if $i \in E_{k}$ or $j \in E_{k}$

(2) $\xi_{i, j}\left(b, b ; E_{k}\right)=I(i=j)$, if $i, j \notin E_{k}$

(3) If $E_{k}=\varnothing$, then $\xi_{i, j}(u, b ; \varnothing)=\xi_{i, j}(u, b)$ is the probability of reaching $b$ in state $j$ prior to ruin given that $J(0)=i$

Let $\xi\left(u, b ; E_{k}\right)=\left(\xi_{i, j}\left(u, b ; E_{k}\right) ; i, j \in E \backslash\left\{E_{k}\right\}\right)$ be an $(m-$ $k) \times(m-k)$ matrix. Using the same arguments as in Section 3 , we can show that $\xi\left(u, b ; E_{k}\right)$ satisfies the following matrixform IDE:

$$
\begin{aligned}
\xi^{\prime}\left(u, b ; E_{k}\right) & =\mathbf{c}_{22}^{-1}\left(\boldsymbol{\lambda}_{22}-\mathbf{A}_{22}\right) \boldsymbol{\xi}\left(u ; E_{k}\right) \\
& =-\mathbf{c}_{22}^{-1} \boldsymbol{\lambda}_{22} \int_{0}^{u} \mathbf{f}_{22}(x) \xi\left(u-x ; E_{k}\right) \mathrm{d} x, \quad 0 \leq u<b,
\end{aligned}
$$

with the boundary condition $\xi\left(b, b ; E_{k}\right)=\mathbf{I}$. It can be shown by using the Laplace transform method that $\mathbf{W}_{22}(u)$ satisfies the IDE in (62) for $0 \leq u<\infty$, we conclude by noting that $\xi\left(b, b ; E_{k}\right)=\mathbf{I}$ that

$$
\xi\left(u, b ; E_{k}\right)=\mathbf{W}_{22}(u)\left[\mathbf{W}_{22}(b)\right]^{-1}, \quad 0 \leq u \leq b .
$$

7.2. Probability of Ruin without the Surplus Hitting a Certain Level $b$ and without Visiting States in $E_{k}$. For $0 \leq u \leq b$ and $i \in E$, we define

$$
\tau_{i}\left(u, b ; E_{k}\right)=\mathrm{P}\left(T_{u}<T_{u}^{b}, J(t) \notin E_{k}, 0 \leq t<T_{u} \mid J(0)=i\right),
$$

to be the probability of ruin without the surplus reaching $b$ and without the process visiting states in $E_{k}$, given that the initial state is $i$. Clearly, we have the following cases:

(1) $\tau_{i}\left(u, b ; E_{k}\right)=0$, if $i \in E_{k}$

(2) $\tau_{i}\left(b, b ; E_{k}\right)=0$, if $i \in E$

(3) $\tau_{i}(u, b ; E \backslash\{i\})$ is the probability of ruin without the surplus hitting a certain level $b$ and without state change from the initial state $i$ prior to ruin

(4) If $E_{k}=\varnothing$, then $\tau_{i}(u, b ; \varnothing)=\tau_{i, j}(u, b)$ is the probability of ruin without the surplus reaching $b$ prior to ruin given that $J(0)=i$

By considering whether or not the surplus has reached $b$ prior to ruin, we have the following formula:

$$
\psi_{i}\left(u ; E_{k}\right)=\tau_{i}\left(u, b ; E_{k}\right)+\sum_{j \notin E_{k}} \xi_{i, j}\left(u, b ; E_{k}\right) \psi_{j}\left(b ; E_{k}\right),
$$

In the matrix form, we have

$$
\vec{\psi}\left(u ; E_{k}\right)=\vec{\tau}\left(u, b ; E_{k}\right)+\xi\left(u, b ; E_{k}\right) \vec{\psi}\left(b ; E_{k}\right),
$$

where $\vec{\tau}\left(u, b ; E_{k}\right)=\left(\tau_{i, j}\left(u, b ; E_{k}\right), i \in E \backslash\left\{E_{k}\right\}\right)^{\top}$. This together with (63) gives

$$
\begin{array}{r}
\vec{\tau}\left(u, b ; E_{k}\right)=\vec{\psi}\left(u ; E_{k}\right)-\mathbf{W}_{22}(u)\left[\mathbf{W}_{22}(b)\right]^{-1} \vec{\psi}\left(b ; E_{k}\right), \\
0 \leq u \leq b .
\end{array}
$$

7.3. Probability of Hitting a Certain Level prior to Ruin without Claims Occurring in States in $E_{k}$. For $0 \leq u \leq b$ and $i, j \in E$, we define $\eta_{i, j}\left(u, b ; E_{k}\right)$ to be the probability of the surplus reaching $b$ in state $j$ without ruin occurring and without claims occurring in states in $E_{k}$, given that $J(0)=i$. Clearly, we have the following cases:

(1) $\eta_{i, j}\left(b, b ; E_{k}\right)=I(i=j)$, if $i, j \in E$

(2) If $E_{k}=\varnothing$, then $\eta_{i, j}(u, b ; \varnothing)=\xi_{i, j}(u, b)$ is the probability of reaching $b$ in state $j$ prior to ruin given that $J(0)=i$

Let $\boldsymbol{\eta}\left(u, b ; E_{k}\right)=\left(\eta_{i, j}\left(u, b ; E_{k}\right) ; i, j \in E\right)$ be an $m \times m$ matrix. Using the same arguments as in Section 5, we can show that $\boldsymbol{\eta}\left(u, b ; E_{k}\right)$ satisfies the following matrix-form IDE:

$$
\begin{aligned}
& \boldsymbol{\eta}^{\prime}\left(u, b ; E_{k}\right)=\mathbf{c}^{-1}(\boldsymbol{\lambda}-\mathbf{A}) \boldsymbol{\eta}\left(u, b ; E_{k}\right) \\
&=-\mathbf{c}^{-1} \lambda\left[\mathbf{I}-\mathbf{I}_{E_{k}}\right] \int_{0}^{u} \mathbf{f}(x) \boldsymbol{\eta}\left(u-x, b ; E_{k}\right) \mathrm{d} x, \\
& 0 \leq u<b,
\end{aligned}
$$

with the boundary condition $\mathbf{\eta}^{\prime}\left(b, b ; E_{k}\right)=\mathbf{I}$.

Using the Laplace transform method, we can show that $\mathbf{V}\left(u ; E_{k}\right)$ satisfies IDE in (68) for $0 \leq u<\infty$. This together with $\boldsymbol{\eta}\left(b, b ; E_{k}\right)=\mathbf{I}$ shows

$$
\boldsymbol{\eta}\left(u, b ; E_{k}\right)=\mathbf{V}\left(u ; E_{k}\right)\left[\mathbf{V}\left(b ; E_{k}\right)\right]^{-1}, \quad 0 \leq u \leq b .
$$

7.4. Probability of Ruin without Hitting a Certain Level $b$ and without Claims Occurring in $E_{k}$. For $0 \leq u \leq b$ and $i \in E$, we define

$$
\zeta_{i}\left(u, b ; E_{k}\right)=\mathrm{P}\left(T_{u}<T_{u}^{b}, N_{u, E_{k}}=0 \mid J(0)=i\right)
$$

to be the probability of ruin without the surplus reaching $b$ and without claims occurring in any state in $E_{k}$ given $J(0)=i$. Clearly, $\zeta_{i}\left(b, b ; E_{k}\right)=0$.

By considering whether or not the surplus has reached $b$ prior to ruin, we have the following formula:

$$
\chi_{i}\left(u ; E_{k}\right)=\zeta_{i}\left(u, b ; E_{k}\right)+\sum_{j \in E} \eta_{i, j}\left(u, b ; E_{k}\right) \chi_{j}\left(b ; E_{k}\right),
$$


TABLE 1: The probability of hitting the target level $b$ prior to ruin without the process visiting certain states.

\begin{tabular}{lcc}
\hline & $b=15$ & $b=20$ \\
\hline$\xi_{1}(u, b ; 2)$ & & 0.000196 \\
$u=0$ & 0.001469 & 0.000398 \\
$u=1$ & 0.002983 & 0.002362 \\
$u=5$ & 0.017706 & 0.017793 \\
$u=10$ & 0.133386 & $1.04995 * 10^{-6}$ \\
\hline$\xi_{2}(u, b ; 1)$ & & $2.29747 * 10^{-6}$ \\
$u=0$ & 0.001132 & 0.000037 \\
$u=1$ & 0.000069 & 0.001107 \\
$u=5$ & 0.001106 & 0.033274 \\
$u=10$ & &
\end{tabular}

TABLE 2: The probability of ruin without the surplus hitting a certain level $b$ and without visiting certain states.

\begin{tabular}{llll}
\hline & $b=15$ & $b=20$ & $b=\infty$ \\
\hline$\tau_{1}(u, b ; 2)$ & & & 0.534611 \\
$u=0$ & 0.534610 & 0.335677 & 0.534611 \\
$u=1$ & 0.335676 & 0.052175 & 0.335677 \\
$u=5$ & 0.052166 & 0.005091 & 0.0052175 \\
$u=10$ & 0.005026 & & 0.338813 \\
\hline$\tau_{2}(u, b ; 1)$ & & 0.243436 & 0.338813 \\
$u=0$ & 0.338813 & 0.064876 & 0.243436 \\
$u=1$ & 0.243436 & 0.012422 & 0.064876 \\
$u=5$ & 0.064873 & & 0.012423 \\
$u=10$ & 0.012343 & &
\end{tabular}

In the matrix form, we have

$$
\vec{\chi}\left(u ; E_{k}\right)=\vec{\zeta}\left(u, b ; E_{k}\right)+\eta\left(u, b ; E_{k}\right) \vec{\chi}\left(b ; E_{k}\right),
$$

where $\vec{\zeta}\left(u, b ; E_{k}\right)=\left(\zeta_{i, j}\left(u, b ; E_{k}\right), i \in E\right)^{\top}$. This together with (69) gives

$$
\begin{array}{r}
\vec{\zeta}\left(u, b ; E_{k}\right)=\vec{\chi}\left(u ; E_{k}\right)-\mathbf{V}\left(u ; E_{k}\right)\left[\mathbf{V}\left(b ; E_{k}\right)\right]^{-1} \vec{\chi}\left(b ; E_{k}\right), \\
0 \leq u \leq b .
\end{array}
$$

\section{Numerical Illustrations}

In this section, we give some numerical results of the probabilities that we have defined in previous sections. We consider two-state Markov-Modulated risk model with intensity matrix as follows:

$$
\mathbf{A}=\left(\begin{array}{rr}
-\frac{1}{4} & \frac{1}{4} \\
\frac{3}{4} & -\frac{3}{4}
\end{array}\right),
$$

and $\pi=\left(\pi_{1}, \pi_{2}\right)=(3 / 4,1 / 4)$. Further assume that claims follow exponential distributions with the following densities:
TABLE 3: The probability of ruin without hitting a certain level $b$ and without claims occurring in certain states with initial state 1.

\begin{tabular}{lccc}
\hline & $b=15$ & $b=20$ & $b=\infty$ \\
\hline$\zeta_{1}(u, b ; 1)$ & & & \\
$u=0$ & 0.054230 & 0.054231 & 0.054231 \\
$u=1$ & 0.039444 & 0.039444 & 0.039444 \\
$u=5$ & 0.011029 & 0.011038 & 0.011038 \\
$u=10$ & 0.002152 & 0.002245 & 0.002247 \\
\hline$\zeta_{1}(u, b ; 2)$ & & & \\
$u=0$ & 0.363240 & 0.363242 & 0.363242 \\
$u=1$ & 0.264192 & 0.264195 & 0.264195 \\
$u=5$ & 0.073910 & 0.073934 & 0.073934 \\
$u=10$ & 0.014803 & 0.015044 & 0.015049 \\
\hline
\end{tabular}

$$
\begin{aligned}
f_{1}(x) & =e^{-x} \\
f_{2}(x) & =0.5 e^{-0.5 x}, \\
x & >0 .
\end{aligned}
$$

Other parameters are set as $\lambda_{1}=1, \lambda_{2}=2 / 3$, $c_{1}=4 / 3$, and $c_{2}=5 / 3$, so that positive loading condition holds true. Then, we have expressions for the probability of ruin without the surplus process visiting certain states and the probability of ruin without a claim occurring in certain states: 


$$
\begin{aligned}
& \psi_{1}(u ; 2)=0.5346 e^{-0.4654 u}, \\
& \psi_{2}(u ; 1)=0.3388 e^{-0.3306 u}, \\
& \chi_{1}(u, 1)=0.0542 e^{-0.3184 u}, \\
& \chi_{1}(u, 2)=0.3632 e^{-0.3184 u}, \\
& \chi_{2}(u, 1)=0.5817 e^{-0.4183 u}, \\
& \chi_{2}(u, 2)=0.2064 e^{-0.4183 u} .
\end{aligned}
$$

Table 1 shows the probability of hitting a target level prior to ruin without the process visiting certain states. Table 2 shows the probability of ruin without the surplus hitting a certain level $b$ and without visiting certain states. Table 3 gives the probability of ruin without hitting a certain level $b$ and without claims occurring in certain states with initial states 1 .

We can see from Table 1 that the probability of hitting a target level prior to ruin without the process visiting certain states increases as initial surplus $u$ increases and but decreases as target level $b$ increases. As the target level increases, it is less likely for surplus process to hit that target level prior to ruin. Table 2 illustrates that the probability of ruin without the surplus hitting a certain level $b$ and without visiting certain states does not change much as the target level changes, but only when initial surplus level is higher, the difference is slightly more obvious. This is because when initial surplus is small, ruin is more likely to occur at an earlier stage. In addition, Table 3 shows that the probability of ruin without hitting a claim occurring in certain states with different initial states performs a similar pattern as shown in Table 2 for similar reasons. At last, when $b$ goes to infinity in Table 2, probabilities become probabilities of ruin without the surplus visiting certain states, and when $b$ goes to infinity in Table 3, probabilities are probabilities of ruin without claims occurring in certain states with initial states 1 .

\section{Concluding Remarks}

In this paper, we study, for a Markov-modulated risk model, some states-specific one-sided exit probabilities and twosided first exit probabilities. These probabilities can be expressed in terms of a modified or a dimension-reduced matrix scale function.

Further research includes the expression of joint and/or marginal Laplace transforms of state-specific occupation time (the duration that the surplus is between an interval and in substate space $E_{k}$ by the time of ruin or other exit times) and the number and the aggregate claims occurred during the state-specific occupation time in terms of the matrixscale functions defined in this paper.

\section{Data Availability}

No data were used to support the findings of this study.

\section{Conflicts of Interest}

The authors declare that they have no conflicts of interest.

\section{Acknowledgments}

Jingchao Li acknowledges the support from the National Natural Science Foundation of China (project no. 11601344) and Shenzhen Peacock Program (project no. 000417).

\section{References}

[1] S. Asmussen, "Risk theory in a Markovian environment," Scandinavian Actuarial Journal, vol. 1989, no. 2, pp. 69-100, 1989.

[2] S. Asmussen, A. Frey, T. Rolski, and V. Schmidt, "Does Markov-modulation increase the risk?" ASTIN Bulletin, vol. 25, no. 1, pp. 49-66, 1995.

[3] N. Bäuerle, "Some results about the expected ruin time in Markov-modulated risk models," Insurance: Mathematics and Economics, vol. 18, no. 2, pp. 119-127, 1996.

[4] H. Schmidli, "Estimation of the Lundberg coefficient for a Markov modulated risk model," Scandinavian Actuarial Journal, vol. 1997, no. 1, pp. 48-57, 1997.

[5] M. Snoussi, "The severity of ruin in Markov-modulated risk models," Bulletin of the Swiss Association of Actuaries, vol. 1, pp. 31-43, 2002.

[6] Y. Lu and S. Li, "On the probability of ruin in a Markovmodulated risk model," Insurance: Mathematics and Economics, vol. 37, no. 3, pp. 522-532, 2005.

[7] Y. Lu, "On the severity of ruin in a Markov-modulated risk model," Scandinavian Actuarial Journal, vol. 2006, no. 4, pp. 183-202, 2006.

[8] A. C. Y. Ng and H. Yang, "On the joint distribution of surplus before and after ruin under a Markovian regime switching model," Stochastic Processes and Their Applications, vol. 116, no. 2, pp. 244-266, 2006.

[9] S. Li and Y. Lu, "Moments of the dividend payments and related problems in a Markov-modulated risk model," North American Actuarial Journal, vol. 11, no. 2, pp. 65-76, 2007.

[10] S. Li and Y. Lu, "The decompositions of the discounted penalty functions and dividends-penalty identity in a Markovmodulated risk model," ASTIN Bulletin, vol. 38, no. 1, pp. 53-71, 2008.

[11] W. Fischer and K. Meier-Hellstern, "The Markov-modulated Poisson process (MMPP) cookbook," Performance Evaluation, vol. 18, no. 2, pp. 149-171, 1993.

[12] J. Li, D. C. M. Dickson, and S. Li, "Some ruin problems for the MAP risk model," Insurance: Mathematics and Economics, vol. 65, pp. 1-8, 2015.

[13] J. Li, D. C. M. Dickson, and S. Li, "Analysis of some ruinrelated quantities in a Markov-modulated risk model," Stochastic Models, vol. 32, no. 3, pp. 351-365, 2016.

[14] J. H. A. De Smit, "The queue GI/M/s with customers of different types or the queue GI/Hm/s," Advances in Applied Probability, vol. 15, no. 2, pp. 392-419, 1983.

[15] S. Li and J. Ren, "The maximum severity of ruin in a perturbed risk process with Markovian arrivals," Statistics \& Probability Letters, vol. 83, no. 4, pp. 993-998, 2013.

[16] B. Chan, H. U. Gerber, and E. S. W. Shiu, "On a classical risk model with a constant dividend barrier," North American Actuarial Journal, vol. 10, no. 2, pp. 133-139, 2006.

[17] N. J. Higham and H. M. Kim, "Numerical analysis of a quadratic matrix equation," IMA Journal of Numerical Analysis, vol. 20, no. 4, pp. 499-519, 2000. 